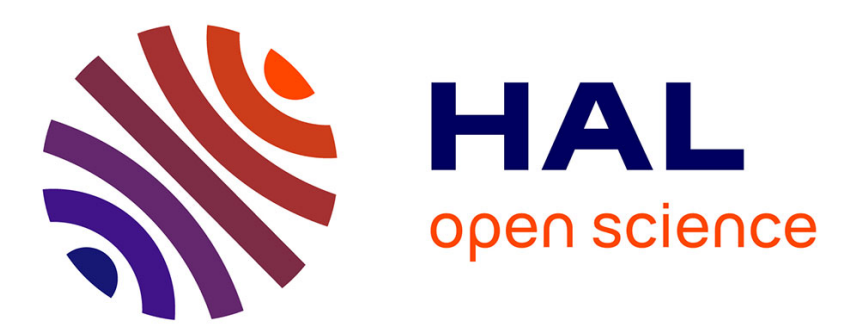

\title{
Excitation and Propagation of Exchange Spin Waves in Ferrite Films with Nonuniformity of Magnetic Parameters across the Film Thickness
}

\author{
A. Temiryazev, M. Tikhomirova, P. Zilberman, A. Maryakhin
}

\section{- To cite this version:}

A. Temiryazev, M. Tikhomirova, P. Zilberman, A. Maryakhin. Excitation and Propagation of Exchange Spin Waves in Ferrite Films with Nonuniformity of Magnetic Parameters across the Film Thickness. Journal de Physique IV Proceedings, 1997, 07 (C1), pp.C1-395-C1-398. 10.1051/jp4:19971160 . jpa-00254806

\section{HAL Id: jpa-00254806 https://hal.science/jpa-00254806}

Submitted on 1 Jan 1997

HAL is a multi-disciplinary open access archive for the deposit and dissemination of scientific research documents, whether they are published or not. The documents may come from teaching and research institutions in France or abroad, or from public or private research centers.
L'archive ouverte pluridisciplinaire HAL, est destinée au dépôt et à la diffusion de documents scientifiques de niveau recherche, publiés ou non, émanant des établissements d'enseignement et de recherche français ou étrangers, des laboratoires publics ou privés. 


\title{
Excitation and Propagation of Exchange Spin Waves in Ferrite Films with Nonuniformity of Magnetic Parameters across the Film Thickness
}

\author{
A.G. Temiryazev, M.P. Tikhomirova, P.E. Zilberman and A.V. Maryakhin* \\ Institute of Radioengineering \& Electronics, Vvedenskii sq. 1, Fryazino, Moscow Region 141120, Russia \\ * Institute of Materials, Gagarin st. 1, 248650 Kaluga, Russia
}

\begin{abstract}
Excitation and propagation of short-wavelength exchange-dominated spin waves in garnet films with nonuniformity of the anisotropy field along the thickness of the film is considered. The paper includes experiments on propagation of pulses of spin waves through the film thickness, and experiments on conversion of spin waves into acoustic waves.
\end{abstract}

\section{INTRODUCTION}

We report on yttrium iron garnet (YIG) films having small relaxation and substantial variation of anisotropy field $H_{a}$ across the film thickness. The films are of interest since they enable efficient linear excitation of short-wavelength exchangedominated spin wave (ESW) propagating through the film thickness. The ESWs with wave number $q$ up to $3 \cdot 10^{5} \mathrm{~cm}^{-1}$ have been excited in our experiments. Such waves can be valuable in applications for two reasons. First, the value of $q$ is large enough for the conditions of synchronism of ESW and acoustic waves (AW) to be satisfied at frequencies as high as $20 \mathrm{GHz}$. Thus, the films can be used for acoustic wave excitation at microwave frequencies. Second, the velocity of ESW is extremely low. A group delay of tens or hundreds of nanoseconds can be achieved under ESW propagation through a thickness of the film, in a distance of a few microns. Therefore, nonuniform films can be used for making small-size microwave delay lines. In addition, in nonuniform films it is possible to obtain any required dependence of time delay on frequency by choosing the right profile of variation in $H_{a}$ along the thickness.

The main difficulty encountered in reaching the ESW application is to obtain an effective conversion from electromagnetic waves to the short-wavelength spin waves. One way of doing this is to use a spatially nonuniform medium $[1,2]$. Because of the nonuniformity the wave number $q$ depends on coordinate. The excitation takes place in a region where $q$ is small, which ensures effective coupling with an electromagnetic wave. As the wave propagates its wavelength decreases, which leads to the appearance of short spin waves. Previously, in experiments $[3,4]$, an inhomogeneity arose due to demagnetization fields existing at the end of the longitudinally magnetized rod. Our main idea is to use nonuniform YIG films, in which an inhomogeneity has been created during the liquid phase epitaxy growth.

The YIG films with thicknesses $L$ from 10 to $30 \mu \mathrm{m}$ were grown epitaxially on gadolinium gallium garnet (GGG) substrates at temperature monotonically varying in time. The variation of the temperature leads to the variation of the uniaxial anisotropy field $H_{a}$ along the $x$-axis perpendicular to the plane of the film. We introduced an effective magnetisation $M$ that was defined as $4 \pi M(x)=4 \pi M_{s a t}(x)-H_{a}(x)$, where $M_{s a t}(x)$ is the saturation magnetisation. The profile of $4 \pi M(x)$ was measured by the processing of spin wave resonance (SWR) spectra $[5,6]$. The films with monotone variation of $4 \pi M(x)$ were used for the experiments in pulsed regime.

\section{PROPAGATION OF ESW PULSES}

The experimental arrangement included a section of $50 \Omega$ strip line of approximately $1 \mathrm{~cm}$ long placed close to an YIG film. We used a microwave switch in order to fed microwave pulses with duration 10-100 ns to this antenna. The pulses reflected from the film were detected, amplified, and displayed on an oscilloscope. For $H=0$, a microwave pulse fed to the setup was totally reflected. Within a certain range of magnetic fields the amplitude of the reflected signal dropped sharply, and a second pulse appeared that was delayed by a time $\tau$ (Fig. 1). The observation of a delayed pulse can be interpreted as evidence for excitation of exchange spin waves that then propagate along the film thickness. Measurements were conducted for two directions of the bias field; $\mathbf{H}$ was directed either perpendicular to the plane of the film (normal field) or in the plane 


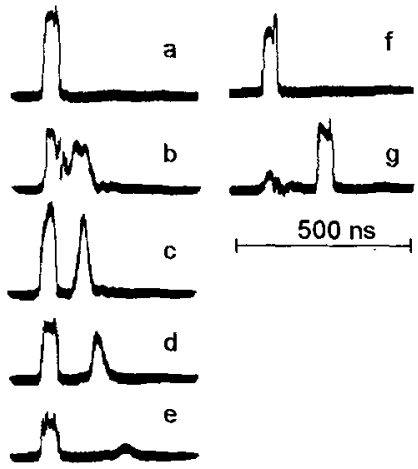

Figure 1: Time dependencies of the envelope of the reflected signal. Traces (a)-(e) are for a tangential field with $\omega / 2 \pi=$ $5124 \mathrm{MHz}$; (a) $-H=0$; (b) $-H=1084 \mathrm{Oe}$; (c) $-H=1079 \mathrm{Oe}$, (d) $-H=1071 \mathrm{Oe},(\mathrm{e})-H=1058 \mathrm{Oe}$. Traces (f) and (g) are for a normal field with $\omega / 2 \pi=5322 \mathrm{MHz} ;(\mathrm{f})-H=0,(\mathrm{~g})-H$ $=3790$ Oe,

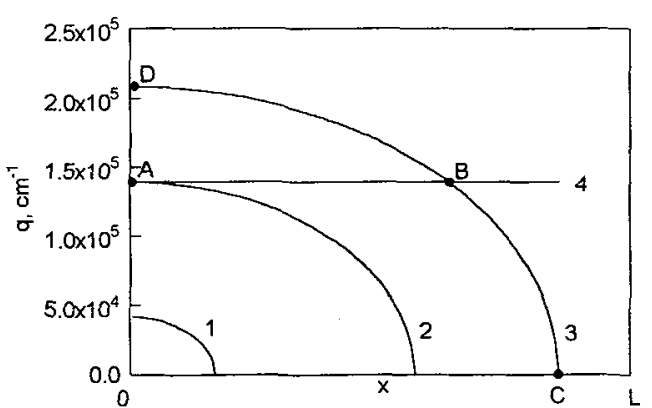

Figure 2: Variation of the wave number along the film thickness. Curves 1-3 show the dependencies $q(x)$ for successive increasing frequency at $H=$ const, or for successive decreasing field magnitude at $\omega / 2 \pi=$ const .

Line 4 - wave number of the acoustic wave

(tangential field). Let us consider the propagation of ESWs in the normal field. Then, the dependence of the local wave number across the thickness of the film has a form

$$
q^{2}(x)=\frac{\omega / \gamma-H+4 \pi M(x)}{D}
$$

where $D=4.6 \cdot 10^{-9}$ Oe $\cdot \mathrm{cm}^{2}$ is the exchange constant. We will assume that $M(x)$ varies monotonically through the thickness of the film, reaching its maximum value $M=M_{0}$ at $x=0$, and its minimum value $M=M_{L}$ at $x=L$. One can see from (1) that if the frequency $\omega$ lies in the range

$$
\omega_{0}<\omega<\omega_{L}
$$

where $\omega_{L}=\gamma\left(H-4 \pi M_{L}\right)$ and $\omega_{0}=\gamma\left(H-4 \pi M_{0}\right)$, then there is a point within the film where the local wave number $q$ turns to zero. We denote by $d$ the coordinate $x$ of that point. The eigenmode is a simusoidal wave (with varying wave number) within the layer $0<x<d$, where $q^{2}>0$, and changes to an exponentially decaying excitation in the region $d<x<L$, where $q^{2}<0$. We will refer to the point $x=d$ as a turning point. The coordinates of the turning point depend both on frequency and on the field magnitude, which is illustrated by Fig. 2, where we show the form of the computed values of $q(x)$ for the case of a parabolic profile $M(x)$. When $\omega \cong \omega_{0}$, the turning point appears near the boundary with large magnetisation $x=0$; as the frequency increases, it shifts into the bulk of the film. When $\omega \cong \omega_{L}$, the value of $d$ reaches the second boundary of the film.

The existence of a turning point within the film causes the exchange spin waves to be excited very efficiently. This latter circumstance is connected with the fact that the of magnetisation varies most smoothly with $x$ near the turning point, giving strong coupling to the external electromagnetic field [7]. Therefore, the layer where $q \sim 0$ acts as an intrinsic spinwave antenna. The delay time is due to the time for propagation of the exchange spin waves from the turning point to the boundary of the film and back. Note that as it is clear from traces (b) to (e) in Fig. 1, the delay time increases as $H$ decreases. At the same time, theoretical considerations based on use of the ESW dispersion law for a uniform medium imply that the ESW group velocity $V_{E S W}$ increases as $H$ decreases, and consequently the delay time $\tau$ must decrease with $H$. The increase in $\tau$, observed in our experiments, is a direct consequence of the nonuniformity of the film properties. The fact is that in a nonuniform film the value of $\tau$ depends not only on $V_{E S W}$ but also on the path length traversed by the exchange spin wave pulse within the film. On this path the condition $q^{2}(x)>0$ is satisfied. Because the coordinate of the turning point changes as the frequency or magnetic field vary, the increase in group velocity can be compensated by an increase in path length, so that $\tau(H)$ will increase. The rate of displacement of the turning point as $H$ or $\omega$ vary is determined by the profile $4 \pi M(x)$. For a given profile, the time delay can be calculated from the relation

$$
\tau(\omega)=\frac{\partial \varphi}{\partial \omega}
$$

where $\varphi$ is the phase shift accumulated by the exchange spin wave as it propagates from the turning point to the boundary of the film and backwards 

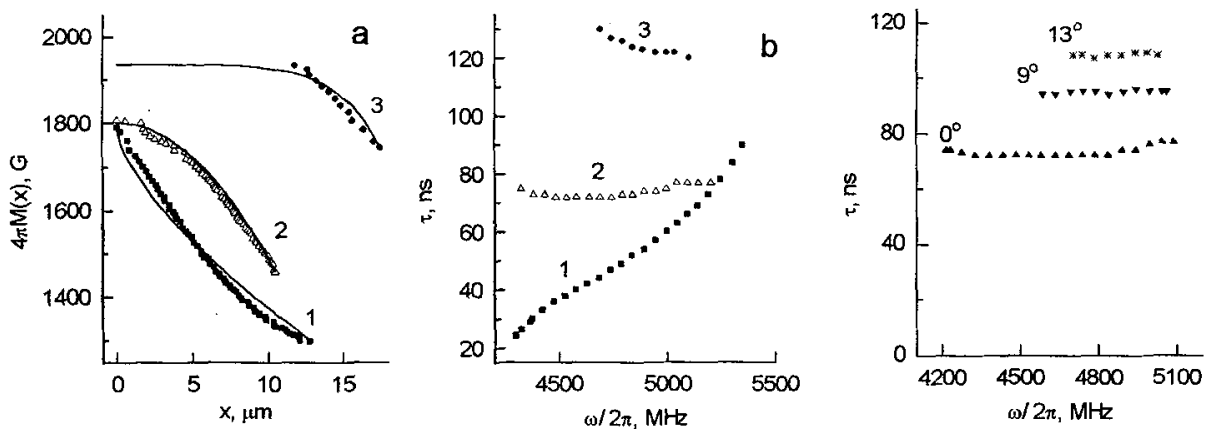

Figure 3: (a) Profiles of effective magnetization variation for three films. The solid curves 1-3 are approximations of the experimental values using power-law functions with exponents $r=2 / 3(1), r=2(2)$, and $r=6$ (3).

(b) Frequency dependencies of the time delay measured on the same films.

(c) Measured frequency dependencies of the time delay as the direction of the external magnetic field is varied. The sample was a YIG film the magnetization profile shown in Fig. 3a, curve 2.

$$
\varphi(\omega, H)=2 \int_{0}^{d\{\omega, H)}|q(x, \omega, H)| d x .
$$

Let us choose $M(x)$ in the form of a power-law function: $4 \pi M(x)=4 \pi M_{0}-4 \pi \Delta M \cdot(x / L)^{r}$. Then for the case of a normally magnetized film, we obtain from (3),(4) and (1) the frequency dependency of $\tau$. When $r=2 / 3$ the delay $\tau$ increases linearly with $\omega$, when $r=2$ the delay time is independent of frequency, and for $r>2$ the delay decreases with increasing frequency. Our experiments confirm these conclusions. Figure 3 shows the profile $4 \pi M(x)$ for three samples, and also the measured functions $\tau(\omega)$. From the figure it is clear that all three basic types of characteristics for $\tau(\omega)$ - increase, decrease, and constancy of the delay - can be realized in practice using films with various profiles. The width of the frequency band (2), in which the delayed pulse is observed, depends on the drop $4 \pi \Delta M=4 \pi M_{0}-4 \pi M_{L}$. It exceeds $1 \mathrm{GHz}$ for the film with $4 \pi \Delta M$ $\approx 500 \mathrm{G}$. From (1) we estimate that the wave numbers of the exchange spin waves in such a sample can be about $3 \cdot 10^{5} \mathrm{~cm}^{-1}$.

Note some features that can be useful for ESW application. First, as it was seen from Fig. $3 b$, when one of the films we investigated was placed in a normal field, it exhibited a roughly constant value of the time delay. Fig. $3 \mathrm{c}$ shows that the absolute value of $\tau$ can be varied within certain limits by applying an external magnetic field $\mathbf{H}$ at a rather small angle $\theta$ to the film normal. Second, it would be useful to study the possibility to shift in frequency the delayed ESW pulse. One way of getting the frequency shift is to vary the magnitude of the external magnetic field in a time while the ESW propagates. We have conducted some experiments aimed to demonstrate the shift of the frequency. Frequency translations up to $30 \mathrm{MHz}$ have been achieved.

We conclude that with the use of nonuniform films we can bring about wide-band excitation of traveling exchange spin wave. This excitation is quite efficient - the overall losses for excitation, propagation, and reception of the exchange spin wave pulses are quite small, about $10 \mathrm{~dB}$, for delay time $\sim 100 \mathrm{~ns}$.

\section{EXCITATION OF ACOUSTIC WAVES}

The magnetoelastic interaction should most strongly manifests itself when spin and acoustic waves are synchronous, i.e., when their wave numbers are equal. Our experiments show that a substantial part of the energy of ESW can be transformed into the energy of the transverse acoustic wave when the condition of synchronism is fulfilled in some layer inside a nonuniform film (point $B$ in Fig. 2). In Fig. 4 a we show the envelope of the pulses reflected from the sample. Two delayed pulses are clearly seen, separated by a time that depends on the value of the external magnetic field. For a certain value of the field, the pulses merge. We may assume that the presence of the additional pulse is connected with excitation of an acoustic wave. The group velocity of ESW is less than one of acoustic wave. Then the signal with the large time delay is naturally associated with the exchange spin wave propagation. The ESW is excited at the turning point (point $C$. in Fig. 2, goes to the film surface (point $D$ in Fig. 2) reflects and goes backwards. The pulse with the small time delay is associated with a wave that propagates as an exchange spin wave on the segment between the turning point and the point of synchronism (segment $C-B$ ). and as an acoustic wave in the layer between the film surface and the point of synchronism (segment $C-D$ ). Knowing the effective magnetization profile of this film, we can compute the phase delay $\varphi$ for these waves and use (3) to find the dependence of the time delay on magnetic field. Comparing the experimental data with the computed data (Fig, $4 b$ ), we confirm this interpretation of the experiment. Note that AW reflects from the film-air surface only. When the film boundary 

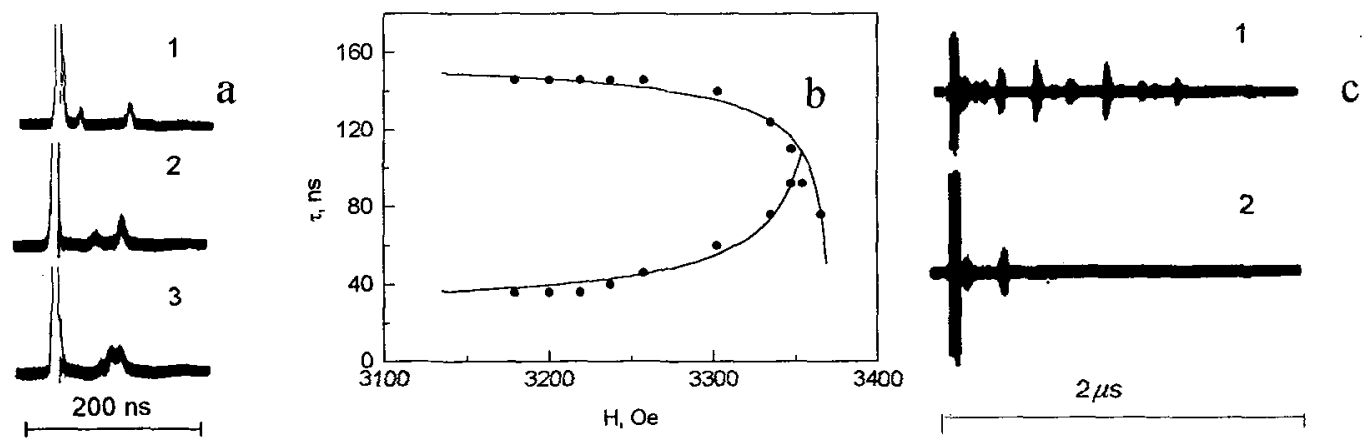

Figure 4: (a) Time dependence of the envelope of reflected signal, $\omega / 2 \pi=3695 \mathrm{MHz}$, normal field; (1) $-H=2933 \mathrm{Oe} ;(2)-H=3018$ $\mathrm{Oe} ;(3)-H=3031 \mathrm{Oe}$.

(b) Dependence of the time delay on magnetic field. The solid curves are computed values of $\tau$, the dots are experimental values

(c) Reflected signal after a heterodyne amplifier. (1) - for a $30 \mu \mathrm{m}$ thick YIG film on $700 \mu \mathrm{m}$ thick GGG substrate, $\omega / 2 \pi=3383 \mathrm{MHz},(2)$

- for the same film but after the surface of the substrate was damaged to make it rough.

$(x=0)$ with the high value of $4 \pi \mathrm{M}$ lies at the film-substrate surface, an acoustic wave penetrates to the interior of substrate, reflects from the second boundary of the substrate and comes back to the YIG film. A typical value of the substrate thickness is $0.5-0.7 \mathrm{~mm}$. A velocity of transverse sound wave in GGG equals to $3.57 \cdot 10^{5} \mathrm{~cm} / \mathrm{s}$. Thus, the acoustic wave comes back in 0.3-0.5 $\mu \mathrm{s}$. The inverse conversion of sound into spin wave may cause the delayed pulse to appear, if the surfaces of the substrate are parallel. The time delay of such pulse should exceed the delay of initial ESW pulse. This is illustrated by Fig. $4 \mathrm{c}$ where we show the reflected signal for a $30 \mu \mathrm{m}$ thick YIG film on $700 \mu \mathrm{m}$ thick GGG substrate.

We estimated the insertion loss for the pulses delayed. It was found that the insertion loss was around $15 \mathrm{~dB}$ when the AW pulse reflected from the film-air surface and it increased up to 23-25 $\mathrm{dB}$ when the elastic wave traveled through the substrate and reflected from its boundary. Since the insertion loss includes the loss for two conversions, as well as transmission loss and reflection loss, we conclude that the loss for AW excitation is less than $10 \mathrm{~dB}$. Thus, nonuniform ferrite films can be used to efficiently excite very high-frequency acoustic waves. Note that a turning point and a point of synchronism should exist simultaneously in order to provide the conversion both from electromagnetic waves into spin waves and from spin waves into acoustic waves. This condition defines the operational band, in which the $\mathrm{AW}$ can be excited

$$
\omega_{s}-\sqrt{\omega_{s}^{2}-2 \omega_{s} \omega_{0}}<\omega<\omega_{L}
$$

where $\omega_{S}=V_{A W}^{2} / 2 \gamma D$, $V_{A W}=3.85 \cdot 10^{5} \mathrm{~cm} / \mathrm{s}$ is the velocity of the transverse acoustic wave in YIG.

It follows from (5) that the maximum frequency of the acoustic wave that can be excited by this way is equal to

$$
\omega_{\text {nax }}=V_{A W} \cdot \sqrt{4 \pi \Delta M / D}
$$

Conditions (5) and (6) shows that if $4 \pi \Delta M=500 \mathrm{G}$ then $\omega_{\max } / 2 \pi \approx 20 \mathrm{GHz}$, and for frequencies $\omega / 2 \pi<9 \mathrm{GHz}$, the bandwidth (5) exceeds $1 \mathrm{GHz}$. Experimentally, we observed AW excitation at frequencies as high as $16 \mathrm{GHz}$ in experiments on SWR in a film with $4 \pi \Delta M=330 \mathrm{G}$. $[6,8]$.

\section{Acknowledgments}

We gratefully acknowledge financial support by the Russian Foundation for Basic Research (Grant No. 94-02-04928-a).

\section{References}

[1]. Schlömann, E., J. Appl. Phys, 35(1964) 159.

[2]. Schlömann, E. and Joseph, R.1., J. Appl. Phys. 35(1964) 2382; Erratum: J. Appl. Phys. 36(1965) 875-876.

[3]. Eshbach, J.R., J. Appl. Phys. 34(1963) Part 2 1298-1304.

[4]. Strauss, W., J. Appl. Phys. 35(1964) 1022.

[5]. Temiryazev A.G., Tikhomirova M.P., and Zil'berman P.E., J. Appl. Phys 76 (1994) 5586-5588.

[6]. Zil'berman P.E., Temiryazev A.G., and Tikhomirova M.P., Sov. JETP 81 (1995) 151-162.

[7]. Gulyaev, Yu.V., Zil'berman, P.E., and Teniryazev, A.G., Sov. Tekh. Phys. Lett. 21 (1995).

[8]. Gulyaev, Yu.V., Temiryazev, A.G., Tikhomirova, M.P., and Zil'berman, P.E., J. Appl. Phys. 75(1994) 5619-5621. 